\title{
A woman presenting with postabortal pyomyoma and treated with exploratory laparotomy with myomectomy and fistulectomy: a case report
}

\author{
Saravanan M. Rajalakshmi ${ }^{1 *}$, Jyoti Priya ${ }^{1}$, Angelica Laiphrakpam ${ }^{2}$, Prof L. Ranjit Singh ${ }^{1}$
}

\author{
${ }^{1}$ Department of Obstetrics and Gynaecology, RIMS, Imphal, Manipur, India \\ ${ }^{2}$ Department of Surgery, RIMS, Imphal, Manipur, India
}

Received: 13 August 2016

Accepted: 12 September 2016

\section{*Correspondence:}

Dr. Saravanan M. Rajalakshmi,

E-mail: rlashwini910@gmail.com

Copyright: $\odot$ the author(s), publisher and licensee Medip Academy. This is an open-access article distributed under the terms of the Creative Commons Attribution Non-Commercial License, which permits unrestricted non-commercial use, distribution, and reproduction in any medium, provided the original work is properly cited.

\begin{abstract}
Pyomyoma (suppurative leiomyoma of the uterus) is a rare condition resulting from infarction and infection of a leiomyoma. It is more usual in pregnant women or postmenopausal women who have vascular disease. The condition is usually fatal unless treated with appropriate antibiotics and surgical intervention. We report a case of a 42-year-old woman who presented with abdominal pain and lump abdomen for 6 months with fever over a period of one month with anaemia for which she was readmitted. She had a history of incomplete abortion with fibroid with red degeneration for which check evacuation was done one and half months before readmission. During the course of treatment in hospital she developed an umbilical fistula with continuous drainage of pus. Her clinical presentation proved to be a diagnostic dilemma mimicking other clinical entities including Tuberculosis and ovarian cancer. Her blood culture and urine culture were sterile. She was widal (dilution) positive and Mantoux negative. Her pus culture from umbilical wound and vaginal swab culture revealed growth of E- coli sensitive to gentamicin and amoxicillin clavulanic acid. An ultrasound scan suggested uterine fibroid with mixed echogenic collection in pelvic cavity with fistulous communication to the umbilicus, but a Magnetic resonance imaging scan suggested a big mass that appeared heterogeneous with fluid filled areas in posterior wall. She was treated with several courses of antibiotics and finally had to undergo laparotomy. During laparotomy, it was found that a large pyomyoma came into view which was successfully removed followed by fistulectomy by surgeon. The patient had uneventful recovery till discharge from hospital. The diagnosis of pyomyoma should be considered in perimenopausal women with large fibroids and pyrexia of unknown origin.
\end{abstract}

Keywords: Postabortal Suppurative Leiomyoma, Fistulectomy, Enteric fever, Multicystic mass, Exploratory laparotomy

\section{INTRODUCTION}

Uterine fibroids are very common, with small leiomyomas present in more than $20 \%$ of women over the age of 40 years, which usually remain asymptomatic. ${ }^{1}$ Thus the recommendation in most women with fibroids in the perimenopausal period is to be conservative. However, large fibroids found in these women may cause infection. ${ }^{2}$ Infection (suppurative leiomyoma of the uterus) is more common in these women because of vascular disease which causes infarction in the fibroids. ${ }^{3}$ Women with large fibroids and co-morbid conditions such as diabetes, who present with pyrexia of unknown origin, should be suspected as having this unusual serious condition until it is proven otherwise. Removal of asymptomatic large fibroids in perimenopausal women 
may therefore be necessary since these patients are not without risk as was previously thought.

\section{CASE REPORT}

The patient was a 42 years old woman who was referred to the Emergency department, Obstetrics and Gynaecology at RIMS with history of pain abdomen, Lump abdomen, numerous bouts of vomiting and fever. She had a history of uterine fibroids with irregular menstrual history with one abortion due to fibroid. Her last sexual activity was before her menses. There was no abdominal or pelvic surgical intervention before her presentation. The provisional diagnosis was fibroid with Enteric fever with anaemia. When examined, it was noted that she was febrile with tachycardia. A non-tender abdominal mass was also noted filling the abdomen arising from pelvic cavity similar to uterus of around 26 weeks size, firm in consistency. Later on she developed an umbilical fistula with variegated consistency of the abdominal mass during further course.

Her blood sugar was (normal $126 \mathrm{mg} / 100 \mathrm{~mL}$ ) and serology for HIV, HBsAg, and HCV were negative. An ultrasound scan revealed a normal gall bladder, kidneys, liver and spleen. A uterus with heterogeneous echo pattern was noted suggesting uterine fibroid. Her fever was not subsiding despite a number of antibiotics. Her blood test results revealed blood sugar of $126 \mathrm{mg} / 100$ $\mathrm{ml}$, haemoglobin $(\mathrm{Hb}) 7.2 \mathrm{~g} / \mathrm{dL}$ (normal range 11-15 $\mathrm{g} / \mathrm{dL}$ ), white blood cell count (WBC) $24.5 \times 109 / \mathrm{L}$ (normal range $4-11 \times 109 / \mathrm{L}$ ) with neutrophilia, platelets $714 \times 109 / \mathrm{L}$ (normal range $150-450 \times 109 / \mathrm{L}$ ) and normal urea and creatinine. Blood and urine cultures were both sterile and she was admitted for intravenous antibiotic treatment. Combination of ceftriaxone, amoxicillin and clavulanic acid; and gentamicin was administered. The patient eventually became afebrile after the course of antibiotic.

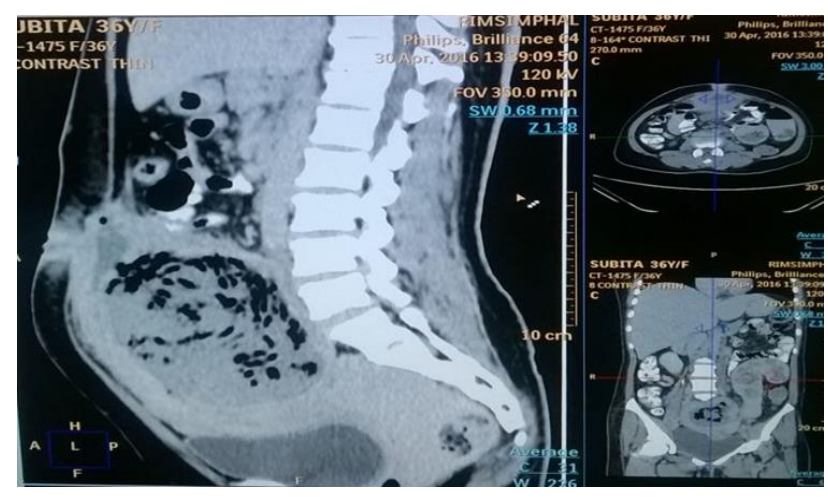

Figure 1: A magnetic resonance imaging scan showing a large multicystic mass $(15.5 \times 16 \times 9 \mathrm{~cm})$ adherent to the abdominal wall.

During her hospital stay, a repeat ultrasound scan revealed a large heterogeneous mass with central cystic areas intimately related to the uterus with a diagnosis of possibly uterine fibroids. A Magnetic resonance imaging (MRI) scan was also done with and without intravenous contrast to evaluate the abdominal mass. This showed a large multicystic mass $15.5 \times 16 \times 9 \mathrm{~cm}$ with communication to umbilicus (Figure 1).

She had 5 PRBC transfusions, 1 iron sucrose transfusion during hospital stay. She was then scheduled for exploratory laparotomy. Intraoperatively myomectomy with fistulectomy was done.

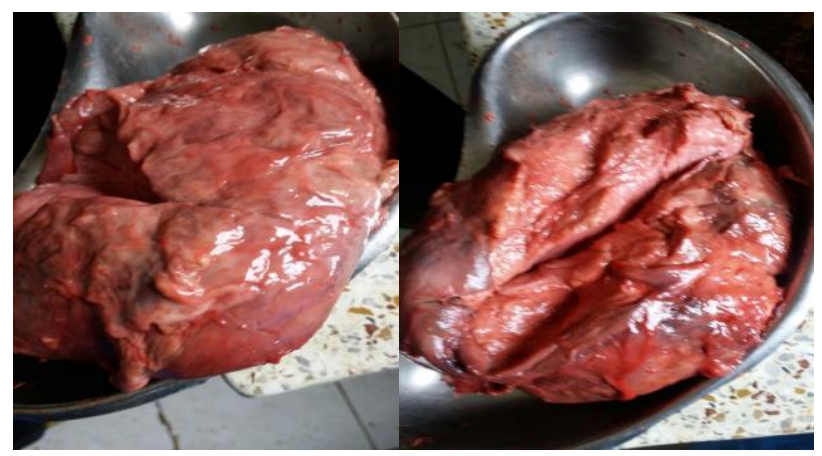

Figure 2: Large posterior fibroid oozing pus.

At exploratory laparotomy, she was found to have a large uterus with a big submucosal fibropid arising from posterior wall of uterus. The uterus was hugely enlarged and boggy in feeling with the infected myoma filling almost the whole of the cavity and was adherent to the anterior abdominal wall and the loops of bowel. By a combination of blunt and sharp dissection, the uterus was freed from the adhesions but in the process, it was inadvertently breached and purulent malodorous fluid (pus) oozed from it. This was suctioned (Figure 2) but not sent for culture and sensitivity. An Exploratory laparotomy with myomectomy and fistulectomy were performed; the surgery was difficult and the patient gravely ill.

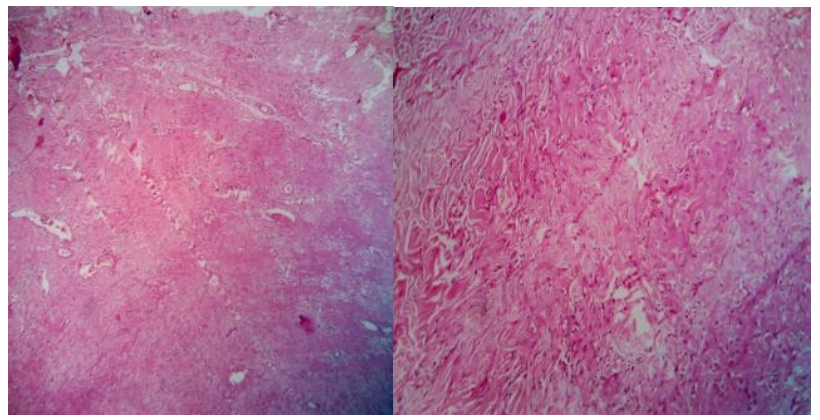

Figure 3: A fibroid showing extensive infarction.

The patient had uneventful post-operative recovery. Treatment with amoxicillin and clavulanic acid were continued post-operatively. On postoperative 5 day she again developed fever and had pus discharge from stitch site. She was started on piperacillin and tazobactum twice daily with local area dressing. After infection control, secondary suturing done and patient was discharged on 
postoperative day 24 and is well at the time of writing. Histological examination of the fibroid showed extensive infarction (Figure 3). The endo metrium was atrophic.

The condition usually results from infarction and infection of a leiomyoma. ${ }^{5}$ Both conditions were clearly evident in this patient. The possible routes of infection for the development of pyomyoma have been described as contiguous spread from the endometrial cavity, direct extension from the adjacent bowel or adnexa, or haematogenous or lymphatic spread from infection elsewhere in the body.

The triad of bacteraemia or sepsis and leiomyoma uteri without any other apparent source of infection should suggest the diagnosis of pyomyoma. ${ }^{7}$ In this case; the patient had sepsis probably due to ascending infection from the vaginal flora. The presence of a large fibroid clinically further substantiated by ultrasonography finding completed the triad.

Diagnosis was made more difficult because of the fact that the fibroid was smeared with thick pus in subsequent ultrasound scan as well as the MRI scan (Figure 1). In one similar case, the patient was perimenopausal and had an intra-uterine device in situ. She was diagnosed as having infected malignant ovarian cancer with an elevated CA 125 level, and was initially treated with broad-spectrum antibiotics; she then underwent total abdominal hysterectomy and bilateral salpingooophorectomy.

Pathological findings showed acute and chronic inflammation of the endometrium with abscess formation in an intramural leiomyoma. ${ }^{3}$ In another case report almost identical to ours, a diabetic post-menopausal woman with a giant pyomyoma simulating an ovarian cancer was described, based on the CT findings of a multicystic mass arising from the pelvis. ${ }^{5}$

In other cases, the causes were illicit drug use causing transient bacteraemia resulting in bacterial seeding of a uterine leiomyoma and instrumentation at dilatation and curettage. $^{8,9}$

In this patient, the presence of chronic pelvic inflammatory disease and along with history of recent dilatation and curettage is suggestive of a spread of infection to the fibroid from the fallopian tubes which was stopped by surgical intervention. Pyomyoma should not be confused with a pyometra and the diagnosis of pyometra is based on the classic symptoms of uterine enlargement, vaginal discharge and acute abdomen. This also requires rapid supportive therapy and surgical intervention. ${ }^{10}$

\section{DISCUSSION}

Pyomyoma (suppurative leiomyoma of the uterus) is a rare disease, which is considered to be a serious complication of uterine leiomyoma. Since 1945 , only 19 patients have been reported in the literature. ${ }^{3}$ It is more usual in pregnant women or postmenopausal women who have vascular disease or with cervical stenosis. ${ }^{2,4}$ Pyomyoma is associated with a high fatality rate of about $21 \% .^{5}$ Only surgical intervention is lifesaving. ${ }^{2}$ The condition may be difficult to diagnose especially in those with a nonspecific clinical presentation without any history of leiomyoma. Delayed diagnosis may result in serious complications, whereas adequate surgery and broad-spectrum antibiotics may decrease serious morbidity and mortality. ${ }^{3}$

The protracted course and myriad differential diagnoses in this patient support this point. The large fibroids, the pyrexia of unknown origin and the classic MRI findings of fibroids with a cystic centre all point to pyomyoma., ${ }^{41}$

\section{CONCLUSION}

This is an excellent teaching case to remind readers of pyomyoma. We hope this case report will help some readers make the diagnosis earlier in their patients.

Funding: No funding sources

Conflict of interest: None declared

Ethical approval: Not required

\section{REFERENCES}

1. Chaparala R, Fawole A, Ambrose N, Chapman A. Large bowel obstruction due to a benign uterine leiomyoma. Gut. 2004;53:386-430.

2. Sah SP, Rayamajhi AK, Bhadani PP. Pyomyoma in a postmeno- pausal woman: a case report. Southeast Asian J Trop Med Public Health. 2005;36:979-81.

3. Manchana T, Sirisabya N, Triratanachat S, Niruthisard S, Tannirandorn Y. Pyomyoma in a perimenopausal woman with intrauterine device. Gynecol Obstet Invest. 2007;63:170-2.

4. Karcaaltincaba M, Sudakoff GS. CT of a ruptured pyomyoma. Am J Roentgenol. 2003;181:1375-7.

5. Genta PR, Dias ML, Janiszewski TA, Carvalho JP, Arai MH, Meireles LP. Streptococcus agalactiae endocarditis and giant pyomyoma simulating ovarian cancer. South Med J. 2001;94:508-11.

6. Mason T, Adair J, Lee Y. Postpartum pyomyoma. J Natl Med Assoc. 2005;97:826-8.

7. Greenspoon JS, Ault M, James BA, Kaplan L. Pyomyoma associated with polymicrobial bacteremia and fatal septic shock: case report and review of the literature. Obstet Gynecol Surv. 1990;45:563-9.

8. Prahlow JA, Cappellari JO, Washburn SA. Uterine pyomyoma as a complication of pregnancy in an intravenous drug user. South Med J. 1996;89:892-5.

9. Das SS, Dogra M, Bala Y. Abscess in fibromyoma following instrumentation. Int $\mathrm{J}$ Gynaecol Obstet. 1994;45:285-7.

10. Blot S, Vandewoude K, Hoste E, Colardyn F. Outcome and attributable mortality in critically Ill 
patients with bacteremia involving methicillinsusceptible and methicillin- resistant Staphylococcus aureus. Arch Intern Med. 2002;162:2229-35.

11. Jones VA, Elkins TE, Wood SA, Buxton BH. Spontaneous rupture of pyometra due to leiomyomata: a case report. J Reprod Med. 1986;31:637-8.
Cite this article as: Rajalakshmi SM, Priya J, Laiphrakpam A, Singh LR. A woman presenting with postabortal pyomyoma and treated with exploratory laparotomy with myomectomy and fistulectomy: a case report. Int J Reprod Contracept Obstet Gynecol 2016;5:3626-9. 